\title{
Association of Serum Magnesium, Copper and Zinc with Severity of Coronary Artery Disease - A Cross-Sectional Study from Salem, India
}

\author{
Philips Abraham ${ }^{1}$, Katherine Quintus Jemy², Sachu Philip ${ }^{3}$, Krishnan Ramalingam \\ ${ }^{1}$ Department of Biochemistry, Al Azhar Medical College and Hospital, Ezhalloor, Thodupuzha, Kerala, India. \\ ${ }^{2}$ Department of Biochemistry, Vinayaka Missions Kirupanandha Medical College, Chinnaseeragapady, Salem, \\ Tamil Nadu, India. ${ }^{3}$ Department of Biochemistry,Vivekanandha Dental College for Women, Tiruchengode, \\ Namakkal, Tamil Nadu, India. ${ }^{4}$ Department of Biochemistry, Narayana Medical College \& Hospital, \\ Chinthareddypalem, Nellore, Andhra Pradesh, India.
}

\section{ABSTRACT}

\section{BACKGROUND}

Coronary artery disease (CAD) remains the principal cause of death and disability in both developed and developing countries. Factors that cause endothelial dysfunction have received increased attention as a potential contributor for atherogenesis. Micronutrients play an important role in maintaining endothelial function as it act as cofactors for number of enzymes. The purpose of this study was to determine plasma level of copper, zinc and magnesium in CAD patients and its correlation with severity of coronary artery disease.

\section{METHODS}

Study group consisted of angiographically positive CAD patients $(n=30)$ and age and sex matched controls $(n=30)$. Severity of the CAD was assessed based on the number, location and percentage of stenosis of coronary vessels involved and SYNTAX score was calculated. CAD patients were classified into grade I, II and III based on SYNTAX score. Level of copper, zinc and magnesium in plasma and hemolysate was analysed using atomic absorption spectrometry. Plasma nitric oxide level was also analysed.

\section{RESULTS}

In our study, Plasma copper level was found to be significantly high and zinc copper ratio, hemolysate Mg level were found to be significantly low in CAD patients. The latter parameters were found to have a significant negative correlation with severity of the diseases and with the level of nitric oxide.

\section{CONCLUSIONS}

Even though a number of confounding factors can influence the copper, zinc and magnesium levels in the body, our study shows that levels of these elements have a significant association with severity of coronary artery diseases.

\section{KEY WORDS}

Coronary Artery Disease, Endothelial Dysfunction, Syntax Score
Corresponding Author:

Dr. Sachu Philip,

140/1A,K.K. Gardens, Mullai Nagar,

Sooramangalam P.O., Salem,

Tamil Nadu, India.

E-mail:philipsachu1@gmail.com

DOI: $10.14260 /$ jemds/2021/662

How to Cite This Article:

Abraham P, Jemy KQ, Philip S, et al. Association of serum magnesium, copper and zinc with severity of coronary artery disease - a cross-sectional study from salem, India. J Evolution Med Dent Sci 2021;10(37):3262-3266, $10.14260 /$ jemds/2021/662

Submission 09-06-2021, Peer Review 24-08-2021 Acceptance 31-08-2021, Published 13-09-2021.

Copyright (C) 2021 Philips Abraham et al. This is an open access article distributed under Creative Commons Attribution License [Attribution 4.0 International (CC BY 4.0)] 


\section{BACKGROUND}

Coronary artery diseases contribute to a higher risk of morbidity and disability in the South Asian population. Deaths from CAD in India have almost doubled in the past 10 years. ${ }^{1}$ CAD involves atherosclerosis of arteries via the slow progression of luminal narrowing. Atherosclerotic plaques are characterized by the storage of apolipoprotein B containing lipoproteins in the space under the endothelium. Lipid accumulation activates dendritic cells, macrophages, smooth muscle cells, and $\mathrm{T}$ cells, leading to maladaptive inflammatory responses. Because dyslipidaemia increases the probability of atherosclerosis, it is a risk factor for CAD. ${ }^{2}$

A study of risk factors in 262 centres from 52 countries in Asia, Europe, the Middle East, Africa, Australia, North America, and South America (among 27,000 Subjects), showed that $90 \%$ of the person attributable risk for CAD can be explained by nine risk factors belonging to the behavioural and physiological groups, namely; low consumption of fruits and vegetables, smoking, alcohol consumption, psychosocial factors, sedentary lifestyle, hypertension, dyslipidaemia, abdominal obesity, and diabetes mellitus. The study also found that the person attributable risk for each of these risk factors not only gets varied by age and sex, but also across geographical regions, which to a great extent was determined by the prevalence of individual risk factors. In South Asians, these risk factors collectively explained $86 \%$ of the total risk. $^{3}$

Normal vascular endothelium, by secreting several mediators including nitric oxide, promotes arterial vasodilation, prevents thrombosis, and has anti-proliferative and anti-inflammatory actions. ${ }^{4}$ A variety of insults like physical injuries, biochemical injury and immune mediated processes may damage endothelial structure and cause endothelial dysfunction. Dysfunction of the endothelium is characterized by impaired vasodilation in response to endothelial-specific agonists that reflects abnormalities in the integrity and function of the vascular endothelium. Dysfunction plays a critical role in the pathogenesis of atherosclerotic coronary artery disease and often precedes development of structural atherosclerosis. ${ }^{5}$

Minerals play an important role in maintaining endothelial function as it act as coenzymes for number of enzymes. Magnesium, the second most abundant cation is a co-factor for over 300 enzymes involved in various metabolism. Even though less than $1 \%$ of total body magnesium is present in the blood, serum level reflects the status of total body content, because an equilibrium is maintained between the body pool. ${ }^{6}$

Since magnesium plays a major role in vascular smooth muscle relaxation, lipid metabolism and proinflammatory response, it has a significant inhibitory role in atherogenesis and incidence of coronary artery disease. ${ }^{7}$ Similarly some studies have reported an association between copper deficiency and hypercholesterolemia, arterial lipid peroxide accumulation and endothelial dysfunction. Low serum level of Zinc - a cofactor for many enzymes and transcription factors, and a mediator of intracellular signals were also found to be associated with increased prevalence of CAD by influencing risk factors. ${ }^{8}$
So the aim of the study was to analyse the association of serum magnesium, copper and zinc with severity of coronary artery disease measured by SYNTAX score.

\section{METHODS}

A cross sectional comparative study was done on 60 subjects aged 40 - 75 years (30 angiographically identified CAD patients and 30 age and sex matched controls). Subjects were selected by simple random technique from the group of patients who were referred to the department of cardiology for coronary angiography and who met the inclusion criteria. Following data were collected during a regular visit to the hospital - anthropometric parameters (including weight, height and waist circumference), history about hypertension, age of onset of diabetes mellitus if present, other chronic disease, medications, use of alcohol, smoking habit, socioeconomic factors, dietary habits as well as the family medical history.

\section{Study Duration}

6 months from January 2015 to june 2015

\section{Ethical and Informed Consent}

The approval from Institutional Ethical Committee was taken (VMKVMC/IEC/15/57) before starting the study. From all patients, a written informed consent was obtained.

\section{Inclusion Criteria}

- $\quad$ Patients with established CAD with vessel disease.

- Patients who had undergone a treadmill test positive for inducible ischemia.

- $\quad$ CAD patients with history of essential hypertension, who had border line rise in fasting blood glucose, and patients with recent onset of diabetes.

\section{Exclusion Criteria}

- Patients excluded were those diagnosed to have coronary artery disease with atrial fibrillation or pacemaker. Patients with history of congestive heart failure and stroke.

- Patients with transient ischemia or carotid surgery or those with history of bypass graft surgery and with transluminal coronary angioplasty.

- Patients with history of peripheral vascular surgery or intermittent claudication.

- Chronic conditions that can influence the body level of minerals like - enteropathies including inflammatory bowel disease, chronic diarrhoea, malabsorption syndrome, renal failure, renal tubular acidosis, hyperthyroidism, hypocalcaemia.

\section{Grouping of Subjects}

- Group I - Comprised of 30 newly diagnosed coronary artery disease patients 
- Group II - Control comprised of 30 healthy subjects.

\section{Baseline Examination}

Standard 12-lead electrocardiogram (ECG) was obtained in supine position and each record was coded independently using the Minnesota Code (MC) by double binding method. 12-lead rest ECGs with abnormal QS pattern (defined as Minnesota codes 4.1 - 2), T - wave inversion (codes 5.1 - 2; T wave inversion $\geq 1.0 \mathrm{~mm}$ ). ${ }^{9}$

\section{Coronary Angiography and Grading of CAD Patients}

Coronary angiography was performed in all subjects with Judkins technique by the cardiologist who was unaware of the current study.

The severity of coronary atherosclerosis was assessed, based on the number of vessels involved. In our study, significant CAD was defined as more than $50 \%$ stenosis in at least one coronary artery segment.

\begin{tabular}{|ccccc|}
\hline & $\begin{array}{c}\text { Severity of Disease } \\
\text { in Angiography }\end{array}$ & $\begin{array}{c}\text { No of Vessels } \\
\text { Involved }\end{array}$ & $\begin{array}{c}\text { Abnormal } \\
\text { QS Pattern }\end{array}$ & $\begin{array}{c}\text { T Wave } \\
\text { Inversion }\end{array}$ \\
$\begin{array}{c}\text { Grade I (mild) } \\
\text { Grade II }\end{array}$ & $<50 \%$ stenosis & Single vessel & 2 leads & 2 leads \\
moderate) & $50-74 \%$ stenosis & Double vessel & 3 - 5 leads & 3 - 5 leads \\
$\begin{array}{c}\text { Grade III } \\
\text { (severe) }\end{array}$ & $75-100 \%$ stenosis & Triple vessel & $>5$ leads & $>5$ leads \\
\hline
\end{tabular}

\section{Sample Collection}

Venous blood sample was collected after an overnight fast of 12 hours from antecubital vein. Serum was used for the estimation of insulin, glucose, total cholesterol, triglycerides, high density lipoprotein (HDL) cholesterol using gold standard methods. Estimation of magnesium, copper and zinc was done by method of atomic absorption spectrometry using atomic absorption spectrometer with graphite furnace (AA7000 Shimadzu). ${ }^{10}$

\section{Statistical Analysis}

All statistical analysis were performed using the SPSS software (Statistical Package for the Social Sciences, version 16.0 SPSS Inc. Chicago, III USA.). Quantitative variables were demonstrated as mean \pm standard deviation. Statistical analysis was done using $t$ test. Association between the factors were analyzed using Pearson's correlation.

\section{RESULTS}

The study was conducted on 30 angiographically defined CAD patients randomly selected from the department of cardiology, Vinayaka Missions Medical College, Salem. According to the percentage of stenosis and involvement of coronary vessels, with guidelines of Minnesota classification, CAD was assessed and severity was classified as mild, moderate and severe.

Values expressed as mean \pm SD. Comparison between two groups were assessed by $\mathrm{t}$ - test. ${ }^{*}$ Statistically significant at $\mathrm{P}$ $<0.05$
Table I depicts the lipid profile level of study subjects. There was significant increase in mean cholesterol level in CAD when compared to control. The mean triglyceride of the normal controls and CAD were $130 \pm 50.77$ and $179.47 \pm$ 90.71 respectively and was found to be significant at $\mathrm{P}<$ 0.001 . There was significant increase in LDL cholesterol level in CAD subjects compared to control at $\mathrm{P}<0.001$.

\begin{tabular}{|ccc|}
\hline Parameters & Control $(\mathbf{N}=\mathbf{3 0})$ & CAD $(\mathbf{N}=30)$ \\
\hline Total cholesterol (mg \%) & $122.8 \pm 10.7$ & $169.45 \pm 47.4^{*}$ \\
Serum triglycerides (mg \%) & $130 \pm 50.77$ & $179.47 \pm 90.71^{*}$ \\
HDL cholesterol (mg \%) & $47.1 \pm 2.9$ & $47.38 \pm 4.6$ \\
LDL cholesterol (mg \%) & $49.6 \pm 11.72$ & $86.17 \pm 42.39^{*}$ \\
VLDL (mg \%) & $26.1 \pm 10.1$ & $35.89 \pm 18.1$ \\
\hline Table 1. Lipid Profile Level of Study Subjects \\
\hline
\end{tabular}

\begin{tabular}{|ccc|}
\hline Parameters & Control $(\mathbf{N}=\mathbf{3 0})$ & CAD $(\mathbf{N}=30)$ \\
Magnesium $(\mathrm{mg} \%)$ & $1.76 \pm 0.349$ & $2.15 \pm 0.257$ \\
Copper $(\mu \mathrm{g} \%)$ & $104.25 \pm 6.66$ & $127.055 \pm 6.028^{*}$ \\
Zinc $(\mu \mathrm{g} \%)$ & $70.86 \pm 2.26$ & $101.75 \pm 2.322^{*}$ \\
\hline Table 2. Biochemical Parameters of Study Subject \\
\hline Statistical analysis was done by students t test.* statistically significant at P $<0.05$ \\
\hline
\end{tabular}

Table II depicts the level of magnesium, copper and zinc in the study subjects. The mean magnesium level of the normal controls, and CAD subjects was not found to be statistically significant while the mean copper and zinc level of the normal controls and CAD were found to be significant at $\mathrm{P}<0.001$.

\begin{tabular}{|cc|}
\hline Parameters & $\begin{array}{c}\text { Severity of CAD } \\
\text { r Value }\end{array}$ \\
\hline Magnesium $(\mathrm{mg} / \mathrm{L})$ & -0.134 \\
Copper $(\mu \mathrm{g} \%)$ & $0.867^{*}$ \\
Zinc $(\mu \mathrm{g} \%)$ & $-0.917^{*}$ \\
\hline Table 3. Correlation of Magnesium, Copper and Zinc with Severity of \\
CAD \\
\hline
\end{tabular}

Table III depicts the results of the Pearson correlation analysis between magnesium, copper and zinc with severity of $\mathrm{CAD}$ in $\mathrm{CAD}$ patients. In CAD subjects, population triglyceride showed significant association with IR $(r=.343$, $\mathrm{P}<0.01$ ) and very low density lipoprotein (VLDL) showed significant correlation with IR. $(\mathrm{r}=.357, \mathrm{P}<0.01)$.

Correlation of magnesium, copper and zinc with lipid profile in CAD subjects. The Pearson's correlation analysis between the total cholesterol, triglyceride, HDL cholesterol, LDL cholesterol with magnesium, copper and zinc in CAD patients showed no significant correlation.

\section{DISCUSSION}

Coronary artery disease is one of the major prevailing non communicable cause of death and disability in the Indian Subcontinent. ${ }^{9}$ It is caused by atherosclerosis, an accumulation of fatty materials on the inner linings of arteries. The occurrence of hyperlipidemia is one of the major etiological factor that contributes to the high incidence of CAD and increased cardiovascular mortality.

Another aspect that influences the occurrence of CAD is nutritive factors which changed considerably with modernization. Studies have revealed that both macro and micro elements like magnesium, copper and zinc has a major role in the maintenance of cardiovascular health. Magnesium as a co-factor for numerous enzymes, is reported to have an 
important role in metabolic process and vascular biology. Magnesium through its involvement in the normal functioning of $\mathrm{Na}+\mathrm{K}+$, ATPase activity, regulates the intracellular level of $\mathrm{Na}$ directly and the level of calcium indirectly. Thus it normalizes peripheral resistance and vasospasm. ${ }^{11}$ Magnesium is also involved in the release of NO from endothelial cell. Thus, magnesium is found to be a converging point of the properties that controls the maintenance of coronary artery. ${ }^{12}$ But in our study, between CAD patients and control subjects, no significant difference was observed in its serum level $90 \%$ of the intracellular magnesium in the body is bound to organic matrices and levels of serum magnesium, represents only $0.3 \%$ of the total content. Serum magnesium levels are further divided into ionized, protein-bound and anion-complexed (i.e. magnesium citrate and phosphate). So the serum level may not accurately reflect the total magnesium status. This might be the reason for the observed insignificant correlation of magnesium level with severity of disease.

Zinc, a trace element that was analysed also has a critical role in redox signal pathway. It has a protective action against activation of oxidative stress (co-factor for antioxidant enzymes) and prevents inflammatory processes to some extent. ${ }^{13}$ When compared to control subjects, we observed a significantly low level of zinc in CAD patients, which agrees with earlier studies. It has been reported that this trace element is involved in the regulation of transcriptional activity of $\mathrm{NF}-\mathrm{kB}$, a controller of apoptosis and inflammation. ${ }^{14}$ By this anti-inflammatory action, Zinc has a protective role in maintaining the integrity of endothelium and reduces the susceptibility to atherosclerosis. ${ }^{15}$ This might be the reason why zinc level showed a negative correlation with the severity of coronary artery disease.

In the current study, we have observed a significantly high level of copper in CAD patients. Some earlier studies have reported the same pattern of observation. ${ }^{16}$ LDL oxidation plays a pivotal role in the process of atherogenesis. Copper is identified to be a very effective catalyst of this process. Moreover, it also upregulates cholesterogenic genes in macrophages, which explains another mechanism for the association of copper and atherosclerosis. These all might have led to the observed positive correlation of copper with severity of disease.

Further studies are required in the following areas of this study to find out erythrocyte or whole blood level which might be able to reflect the functional status of magnesium and also whether the blood level of these minerals have an diagnostic or prognostic value in cardiovascular diseases.

Minerals have a major role in vascular biology and it has a protective role in the maintenance of endothelial integrity. Serum magnesium level is only around $3 \%$ of total body content and exist in different form. So it does not reflect the total body status and cannot be used to correlate with functional status of magnesium. To overcome this, erythrocyte magnesium level or tissue magnesium level may be estimated. Zinc and copper had shown a strong correlation with the severity of disease. So, during fixing the treatment plan for CAD patients and high risk patients, dietary or therapeutic intervention to restore its level should also be included considered. Prognostic and diagnostic values of these minerals can be confirmed by extending the study on a large population.

\section{CONCLUSIONS}

In this study, no significant difference was observed in the serum magnesium level between CAD subjects and control while zinc level was found to be low in CAD compared to control subjects. A significantly high level of copper was observed in the CAD subjects when compared to the control.

Data sharing statement provided by the authors is available with the full text of this article at jemds.com.

Financial or other competing interests: None.

Disclosure forms provided by the authors are available with the full text of this article at jemds.com.

The authors would like to acknowledge the Indian Council Of Medical Research, for accepting this study and for the coverage of financial support. Also we acknowledge Dr Ramalingam for providing the facilities to carry out the study.

\section{REFERENCES}

[1] Kavi A, Walvekar PR, Patil RS. Biological risk factors for coronary artery disease among adults residing in rural area of North Karnataka, India. Journal of Family Medicine and Primary Care 2019;8(1):148-53.

[2] Mangge $\mathrm{H}$, Almer G. Immune-mediated inflammation in vulnerable atherosclerotic plaques. Molecules 2019;24(17):3072.

[3] Yusuf S, Hawken S, Ounpuu S, et al. Effect of potentially modifiable risk factors associated with myocardial infarction in 52 countries (the INTERHEART study): case - control study. Lancet 2004;364(9438):937-52.

[4] Cai H, Harrison DG. Endothelial dysfunction in cardiovascular diseases: the role of oxidant stress. Circulation Research 2000;87(10):840-4.

[5] Ormazabal V, Nair S, Elfeky 0, et al. Association between insulin resistance and the development of cardiovascular disease. Cardiovascular Diabetology 2018;17(1):122.

[6] Chakraborti S, Chakraborti T, Mandal M, et al. Protective role of magnesium in cardiovascular diseases: a review. Molecular and Cellular Biochemistry 2002;238(1-2):16379.

[7] Kupetsky-Rincon EA, Uitto J. Magnesium: novel applications in cardiovascular disease-a review of the literature. Annals of Nutrition and Metabolism 2012;61(2):102-10.

[8] Torkanlou K, Bibak B, Abbaspour A, et al. Reduced serum levels of zinc and superoxide dismutase in obese individuals. Annals of Nutrition and Metabolism 2016;69(3-4):232-6.

[9] Prineas RJ, Crow RS, Zhang ZM. The Minnesota code manual of electrocardiographic findings. Springer Science \& Business Media, 2009.

[10] Perkin-Elmer. Analytical methods for atomic absorption spectroscopy. U.S.A.: Perkin - Elmer Corp., 1982: p. 1300.

[11] Burguera JL, Burguera M, Rondon C, et al. Determination of beryllium in natural and waste waters using on - line flow-injection preconcentration by precipitation/ dissolution for electrothermal atomic absorption spectrometry. Talanta 2000;52(1):27-37. 
[12] Kostov K, Halacheva L. Role of magnesium deficiency in promoting atherosclerosis, endothelial dysfunction, and arterial stiffening as risk factors for hypertension. International Journal of Molecular Sciences 2018;19(6):1724.

[13] Roth HP, Kirchgessner M. Zinc and insulin metabolism. Biological Trace Element Research 1981;3(1):13-32.

[14] Mussbacher M, Salzmann M, Brostjan C, et al. Cell type specific roles of $\mathrm{NF}-\kappa \mathrm{B}$ linking inflammation and thrombosis. Frontiers in Immunology 2019;10:85.
[15] Chernyavskiy I, Veeranki S, Sen U, et al. Atherogenesis: hyperhomocysteinemia interactions with LDL, macrophage function, paraoxonase 1 and exercise. Annals of the New York Academy of Sciences 2016;1363(1):138-54.

[16] Tsoukatos DC, Arborati M, Liapikos T, et al. Copper catalyzed oxidation mediates PAF formation in human LDL subspecies: protective role of PAF: acetylhydrolase in dense LDL. Arteriosclerosis, Thrombosis and Vascular Biology 1997;17(12):3505-12. 